\title{
Exciton energies and wave functions in hexagonal Boron Nitride using Miller and Good's uniform approach
}

\author{
J. C. G. Henriques • N. M. R. Peres
}

Received: date / Accepted: date

\begin{abstract}
In this paper we revisit the work of Miller and Good, which describes an uniform JWKB type of approximation to the solution of quantum problems. This paper, very well known in atomic physics in the 70's 80's of the last century, did not attract the same attention from the condensed matter community. Contrary to the usual JWKB approach, Miller and Good's method yields wave functions that do not diverge at the classical turning points. We apply the method in the context of two-dimensional excitons, an important condensed matter system. In particular, we apply our results to excitons in hexagonal boron nitride, solving the corresponding Wannier equation. We compare the semiclassical results with others from the literature and find good agreement.
\end{abstract}

Keywords JWKB $\cdot 2 \mathrm{D}$ Materials $\cdot$ Excitons

\section{Introduction}

The JWKB approximation, after Jeffreys, Wentzel, Kramers, and Brillouin $[1-3]$ (see also $[4,5]$ ) is commonly used as an approximated method to find the eigenvalues of a given one-dimensional potential and to determine the tunnelling probability of a particle through a barrier. This is particularly useful for potentials where analytical solutions are non-existent. The corresponding

\footnotetext{
N. M. R. Peres

Department and Centre of Physics, and QuantaLab, University of Minho, Campus of Gualtar, 4710-057, Braga, Portugal

International Iberian Nanotechnology Laboratory (INL), Av. Mestre José Veiga, 4715-330, Braga, Portugal

E-mail: peres@fisica.uminho.pt

J. C. G. Henriques

Department and Centre of Physics, and QuantaLab, University of Minho, Campus of Gualtar, 4710-057, Braga, Portugal
} 
wave functions, on the other hand, are known to be problematic, and require the use of special functions for analysing their behaviour in the vicinity of the classical turning points, where the total energy equals the potential energy. Moreover, the JWKB wave functions suffer from a major drawback: they diverge at the classical turning points. Therefore, they are not uniform across the spatial range of the real axis. Although this sounds like a severe limitation, they still find their use in applications [6-9].

In order to overcome this difficulty, Miller and Good [10,11] developed an uniform description, in the same spirit of the JWKB approximation, that does not suffer from divergences at the classical turning points. In their approach, the wave function is a product of two spatial dependent functions. One of the functions, $A(x)$, is explicitly dependent on the position, whereas the second function, $\phi[\xi(x)]$, depends on $\xi(x)$, which has an implicit dependence on the position. The function $\phi(\xi)$ obeys a differential equation termed the comparison equation, and whose solution is known. The function $A(x)$ plays the same role as the amplitude of the JWKB, with the extra bonus of being regular at the classical turning points. The semiclassical approximation appears when the unknown function $\xi(x)$ is expanded in positive powers of the reduced Planck constant. In a subsequent publication [12], Good extended the method to the solution of the three dimensional Schrodinger equation with radial symmetry. The extension to two dimensions was made by Berry and Ozorio de Almeida [13].

In this paper we revisit the method of Miller and Good in the context of the calculation of energy levels and wave functions of two-dimensional (2D) excitons [14]. This class of excitons has been found in two dimensional semiconductors, such as transition metal dichalcogenides (TMDs) [14,15], and insulators, like hexagonal boron nitride (hBN) [16-18], and is currently a subject of great interest. Due to its simplicity, we will focus our study on the hBN, but the extension to TMDs is not difficult.

Excitons are bound states of electrons and holes created by the interaction of the electrons in the valence band with an external electromagnetic field. If the energy of the incoming photon is of the order of the band gap, an electron is removed from the valence band and placed in the conduction band, leaving a hole behind. The created electron and the hole have opposite charges and can bind together via the electrostatic interaction, thus creating a kind of a "hydrogen atom" inside a solid.

Two dimensional excitons were recently discovered in 2D-materials [14]. They show much larger binding energies than their counterparts in three dimensional semiconductors due to their reduced dimensionality. Indeed, since $2 \mathrm{D}$ materials are essentially a surface and the electrostatic interaction is three dimensional, most of the field lines lie outside the material and are, therefore, not screened, thus leading to larger binding energies. In addition, and at odds with excitons in three dimensions, the electrostatic interaction between the two opposite charges in the exciton is not the usual Coulomb interaction but is described rather by the Rytova-Keldysh potential [20,21]. 
The goal of the paper is twofold: (i) to revisit the method of Miller and Good, and (ii) to determine the binding energy of the excitons and their wave functions, which results from the solution of the Wannier equation. The latter is a highly desirable result, since accurate binding energies are often only obtained trough computationally demanding approaches [22].

The paper is organized as follows: in Sec. II, we revisit the Miller-Good uniform method in one dimension. This sets the stage to the two-dimensional case. In Sec. III, we extend the method to two-dimensions and apply it to the case of the 2D Hydrogen atom, where we recover the known analytical solution for the binding energies. In Sec. IV, we apply the Miller-Good method to the solution of Wannier equation describing excitons in 2D-materials. Finally, in Sec. $\mathrm{V}$ we give the concluding remarks.

\section{The uniform method of Miller and Good in one dimension}

In this section we introduce the uniform method of Miller and Good in one dimension. This approach consist, as does the JWKB, in an expansion of a certain function in powers of $\hbar$, the reduced Planck's constant. On the other hand, and contrary to the JWKB, the amplitude of the wave function is well defined throughout the whole real axis. The introduction of a comparison differential equation allows one to solve the problem near the classical turning points. This treatment, as we shall see, removes the divergence of the wave function at the classical turning points that are known to appear when the JWKB is used. A similar method, based solely on Airy's function, was applied with success to the description of non-homogeneous wave guides [23,24].

The problem we aim to address is the solution of the eigenvalue problem defined by the Schrodinger equation:

$$
\frac{d^{2}}{d x^{2}} \psi(x)+\frac{2 m}{\hbar^{2}}[E-V(x)] \psi(x)=0
$$

where $m$ is the mass, $E$ is the energy and $V(x)$ is the potential. It is well known that the JWKB [3] wave function can be written as:

$$
\psi_{\mathrm{JWKB}}(x)=\frac{C}{\sqrt[4]{p^{2}(x)}} \exp \left[ \pm i \int^{x} d x^{\prime} \sqrt{p^{2}\left(x^{\prime}\right)} / \hbar\right],
$$

where $p(x)$ reads:

$$
p(x)=\sqrt{2 m[E-V(x)]},
$$

and $C$ is some constant. It is evident that at the classical turning points, defined from the condition $E-V\left(x_{c}\right)=0$, the amplitude of the JWKB wave function diverges. The method of Miller and Good copes with this difficulty, defining a new starting point for the wave function. We now turn to a detailed presentation of the method. 
To start developing the Miller and Good's method, we write the wave function as:

$$
\psi_{\mathrm{MG}}(x)=A(x) \phi[\xi(x)],
$$

where $\xi(x)$, as we will shortly see, is an implicit function of the spatial coordinate. Now, an auxiliary problem, termed comparison equation, with an established solution is introduced:

$$
\frac{d^{2}}{d \xi^{2}} \phi(\xi)+\frac{\kappa^{2}(\xi)}{\hbar^{2}} \phi(\xi)=0
$$

where $\kappa(\xi)$ is a known function, that should be chosen as close as possible to $p(x)$ (we will see that this requirement is not as stringent as it sounds). Inserting Eq. (4) in Eq. (1), using the auxiliary problem and finally demanding that the term proportional to $\phi^{\prime}[\xi(x)]$ must vanish we arrive at the function $A(x)$. In a more detailed manner, inserting Eq. (4) in Eq. (1) yields:

$$
\begin{aligned}
& A^{\prime \prime}(x) \phi[\xi(x)]+\phi^{\prime}[\xi(x)]\left[2 A^{\prime}(x) \xi^{\prime}(x)+A(x) \xi^{\prime \prime}(x)\right]+A(x) \xi^{\prime}(x)^{2} \phi^{\prime \prime}[\xi(x)] \\
+ & \frac{p^{2}(x) A(x) \phi[\xi(x)]}{\hbar^{2}}=0
\end{aligned}
$$

where the primes denote differentiation relative to $x$. Using the differential equation for $\phi$, the comparison equation, we eliminate $\phi^{\prime \prime}$ from the previous expression:

$$
\begin{aligned}
& A^{\prime \prime}(x) \phi[\xi(x)]+\phi^{\prime}[\xi(x)]\left[2 A^{\prime}(x) \xi^{\prime}(x)+A(x) \xi^{\prime \prime}(x)\right]-\frac{A(x)\left[\xi^{\prime}(x)\right]^{2} \kappa^{2}[\xi(x)] \phi[\xi(x)]}{\hbar^{2}} \\
+ & \frac{p^{2}(x) A(x) \phi[\xi(x)]}{\hbar^{2}}=0 .
\end{aligned}
$$

The term proportional to $\phi^{\prime}[\xi(x)]$ can also be eliminated if one demands that

$$
2 A^{\prime}(x) \xi^{\prime}(x)+A(x) \xi^{\prime \prime}(x)=0 .
$$

This requirement implies:

$$
A(x)=\frac{1}{\sqrt{\xi^{\prime}(x)}} .
$$

Thus, the proposed wave function may be written as:

$$
\psi_{\mathrm{MG}}(x)=\frac{1}{\sqrt{\xi^{\prime}(x)}} \phi[\xi(x)]
$$

where $\xi(x)$ is yet to be determined. Up to this point the calculation is exact. Now, we assume, similarly to the JWKB approach, that the function $\xi(x)$ can be expressed as a power series. Since Eq. (7) only depends on even powers of $\hbar$ we write:

$$
\xi(x)=\xi_{0}(x)+\xi_{2}(x)+\xi_{4}(x)+\ldots
$$


where the sub-indexes refer to powers of $\hbar$. Considering only the lowest order term in $\xi(x)$, that is $\xi(x) \approx \xi_{0}(x)$, and neglecting the second derivative of $A(x)$ (since it will clearly be associated with higher order terms in $\hbar$ ), we find:

$$
-\left[\xi_{0}^{\prime}(x)\right]^{2} \kappa^{2}[\xi(x)]+p^{2}(x)=0,
$$

from where we obtain:

$$
\xi_{0}^{\prime}(x)=\sqrt{\frac{p^{2}(x)}{\kappa^{2}\left[\xi_{0}(x)\right]}} .
$$

Therefore, the approximate solution to the Schrodinger equation reads:

$$
\psi_{\mathrm{MG}}(x)=\left(\frac{\kappa^{2}[\xi(x)]}{p^{2}(x)}\right)^{1 / 4} \phi[\xi(x)],
$$

where $\kappa, p$ and $\phi$ are known, and $\xi$ is yet to be determined. This expression clearly resembles that of the JWKB, however, in this case, $\kappa^{2}[\xi(x)]$ is not a constant, which is key aspect in making the wave function regular at the classical turning points (note that in the JWKB approach, $\kappa^{2}=1$ ). The remaining task is to find $\xi(x)$. This is readily done solving Eq. (13), that is

$$
\int_{\xi_{c}}^{\xi(x)} \sqrt{\kappa^{2}\left(\xi^{\prime}\right)} d \xi^{\prime}=\int_{x_{c}}^{x} \sqrt{p^{2}\left(x^{\prime}\right)} d x^{\prime},
$$

where $\xi_{c}=\xi\left(x_{c}\right)$ is one of the classical turning points of $\kappa$, that is $\kappa\left(\xi_{c}\right)=0$. The choice of the lower integration limit guarantees that the wave function is finite at the classical turning point, since when $p^{2}(x)$ vanishes so does $\kappa^{2}[\xi(x)]$; strictly speaking we get an indeterminacy which becomes finite in the sense of a limit. This is the main advantage of the Miller and Good approach in comparison with the usual JWKB.

\section{The radial Schrodinger equation and the 2D Hydrogen atom}

In this section we extend the methods of the previous one to problems with radial symmetry in two dimensions. We will start by writing the Schrodinger equation for the case of the 2D Hydrogen atom, from which a differential equation defining the radial component of the wave function is obtained. Then, the Miller and Good method presented in the previous section is applied.

The starting point is, once again, the Schrodinger equation, which, in atomic units (a. u.), reads:

$$
\nabla^{2} \psi(r, \theta)+2[E-V(r)] \psi(r, \theta)=0
$$

where $E<0$ is the energy, $V(r)$ is the Coulomb potential (chosen to illustrate the method for its importance):

$$
V(r)=-\frac{1}{r}
$$


and $\nabla^{2}$ is the two dimensional Laplacian:

$$
\nabla^{2}=\frac{\partial^{2}}{\partial r^{2}}+\frac{1}{r} \frac{\partial}{\partial r}+\frac{1}{r^{2}} \frac{\partial^{2}}{\partial \theta^{2}}
$$

We now propose a wave function with separate radial and angular contributions, that is:

$$
\psi(r, \theta)=R(r) e^{i m \theta},
$$

where $m$ is the angular quantum number. Since we will only be concerned with $s$-states, that is states with $m=0$, the wave function will only depend on the radial coordinate. Plugging Eq. (19), with $m=0$, into Eq. (16) we obtain:

$$
\frac{d^{2}}{d r^{2}} R(r)+\frac{1}{r} \frac{d}{d r} R(r)+2\left(E+\frac{1}{r}\right) R(r)=0 .
$$

Traditionally, one would now introduce a variable change of the form $R(r) \sqrt{r}=$ $P(r)$ and produce a new differential equation where the terms containing a first derivative in $r$ vanish. This change is equivalent to shifting the focus from the probability density $R(r)$ to the probability distribution $P(r)$, which are equivalent descriptions of the same problem. However, it is know that when $P(r)$ is used in a JWKB frame of work, problems appear. These problems prompted Kramers to introduce an ad hoc modification to the potential, and Langer to introduce a variable change (the Langer transformation) that produced the desired results. Recently, it has been shown [26] that both of these approaches are not necessary if one uses $R(r)$ instead of $P(r)$. In fact, the authors show that a JWKB type of approach is always possible, in any dimension, as long as the correct form of the differential equations is used. Moreover, Ref. [26] also points out that the Langer transformation is nothing more than an alternative representation of the problem when $R(r)$ is used (which justifies the correct results obtained by Langer). Although not strictly necessary based on the aforementioned reasons, we will follow Langer's approach since it allows us to make an easier transition from the formalism presented in the previous section to the present case of study.

Defining the radial function as $R(r)=P(r) / \sqrt{r}$, the previous equation simplifies to:

$$
\frac{d^{2}}{d r^{2}} P(r)+\left(2 E+\frac{2}{r}+\frac{1}{4 r^{2}}\right) P(r)=0 .
$$

This one dimensional differential equation resembles the one used in the previous section when the Miller and Good formalism was introduced. Thus, one may be tempted to promptly apply this method to Eq. (21). However, as was pointed before, in its current form, this equation is troublesome. To deal with this problem, we introduce Langer's transformation, defined as:

$$
\begin{aligned}
r & =e^{x}, \\
P(r) & =e^{x / 2} X(x) .
\end{aligned}
$$

The introduction of these definitions removes the singularities at the origin and extends the domain of the problem across the whole real axis. Historically, this 
was the reason that Langer considered to be at the origin of problems he found. Explicitly introducing this transformation in Eq. (21) we obtain:

$$
X^{\prime \prime}(x)+\left(2 E e^{2 x}+2 e^{x}\right) X(x)=0 .
$$

Note that $\exp (x / 2) \rightarrow \sqrt{r}$, and thus describing the problem with $X(x)$ is nothing more than describing it with $R(r)$ with a transformed variable. Although historically motivated by wrong ideas, the Langer transformation produces a correct mathematical description of the problem. With Eq. (24) we can promptly apply the Miller and Good approach to solve the 2D Hydrogen atom problem.

In order to solve Eq. (24), we start by noting that the function $2 E e^{2 x}+2 e^{x}$ in Eq. (24) vanishes at the classical turning point:

$$
x_{c}=\ln \left(-\frac{1}{E}\right) \text {. }
$$

The other classical turning point where the potential term vanishes occurs at the origin, which in this new coordinate system corresponds to $x \rightarrow-\infty$. These two turning points should be treated separately. To solve the problem near the origin we note that in the limit $r \rightarrow 0$ the solution to Eq. (20) is a superposition of Bessel functions. Inspired by this behaviour we introduce the following auxiliary equation [13]:

$$
\frac{d^{2}}{d \xi^{2}} \phi(\xi)+2 e^{\xi} \phi(\xi)=0
$$

which has a turning point when $\xi \rightarrow-\infty$ and whose solution reads:

$$
\phi(\xi)=c_{1} J_{0}\left(2 \sqrt{2} \sqrt{e^{\xi}}\right)+c_{2} Y_{0}\left(2 \sqrt{2} \sqrt{e^{\xi}}\right)
$$

where $J_{0}(x)$ and $Y_{0}(x)$ are Bessel functions of the first and second kind, respectively. Since only $J_{0}(x)$ is finite at $x=0$, we write $X(x)$ as:

$$
\phi(\xi)=c_{1} J_{0}\left(2 \sqrt{2} \sqrt{e^{\xi}}\right) .
$$

According to the Miller and Good approach, the solution near the origin reads:

$$
X_{1}(x)=\left(\frac{2 e^{\xi(x)}}{\left|2 E e^{2 x}+2 e^{x}\right|}\right)^{1 / 4} J_{0}\left(2 \sqrt{2} \sqrt{e^{\xi(x)}}\right),
$$

where the function $\xi(x)$ is obtained from the relation:

$$
\begin{aligned}
\int_{-\infty}^{\xi(x)} \sqrt{2} e^{\xi(x) / 2} d \xi & =\int_{-\infty}^{x} \sqrt{2 E e^{2 x}+2 e^{x}} d x \\
2 \sqrt{2} e^{\xi(x) / 2} & =e^{x / 2} \sqrt{2 E e^{x}+2}+\frac{2}{\sqrt{-2 E}} \arcsin \left(\frac{e^{x / 2} \sqrt{-2 E}}{\sqrt{2}}\right)
\end{aligned}
$$


Now, we move on to solve the problem in the vicinity of the classical turning point $x_{c}=\ln (-1 / E)$. To do so, we introduce the Airy equation as the comparison equation:

$$
\frac{d^{2}}{d \xi^{2}} \phi(\xi)-\xi \phi(\xi)=0
$$

which has a turning point at $\xi=0$ and whose solution is obviously:

$$
\phi(\xi)=c_{2} \operatorname{Ai}(\xi) .
$$

The choice of the Airy equation as the comparison equation is based on its simplicity and, as we will see, the quality of the results. Some intuition regarding this choice can be gained if one expands the term inside the parenthesis in Eq. (20) about the respective classical turning point. Once this is done, the Airy equation (and another two terms) appears. Once again, using the Miller and Good approach, we write:

$$
X_{2}(x)=\left(\frac{|\xi(x)|}{\left|2 E e^{2 x}+2 e^{x}\right|}\right)^{1 / 4} \operatorname{Ai}[\xi(x)],
$$

where the function $\xi(x)$ is given by:

$$
\int_{\xi(x)}^{0} \sqrt{-\xi} d \xi=\int_{x}^{x_{c}} \sqrt{2 E e^{2 x}+2 e^{x}} d x .
$$

At this point, we note that $\xi(x)$ has different definitions above and bellow the classical turning point, since the term inside the square root on the right hand side will have different signs depending on where $x$ sits relatively to $x_{c}$. For $x<x_{c}$ we have:

$$
\xi(x)=-\frac{3}{2}\left[-e^{x / 2} \sqrt{2 E e^{x}+2}-\frac{2}{\sqrt{-2 E}} \arcsin \left(\frac{e^{x / 2} \sqrt{-2 E}}{\sqrt{2}}\right)+\frac{\pi}{\sqrt{-2 E}}\right]^{2 / 3},
$$

and for $x>x_{c}$ :

$$
\xi(x)=-\frac{3}{2}\left[e^{x / 2} \sqrt{-2 E e^{x}-2}+\frac{2}{\sqrt{-2 E}} \operatorname{arcsinh}\left(\frac{e^{x / 2} \sqrt{2 E}}{\sqrt{2}}\right)-\frac{\pi}{\sqrt{2 E}}\right]^{2 / 3} .
$$

Now, we observe that the obtained wave functions will only generate the physically correct solutions for specific values of $E$, that is, for the eigenvalues of the Schrodinger equation. In order to obtain these energies a quantisation condition must be imposed. To obtain this condition, and thus the relevant eigenenergies, we study the asymptotic behavior of $X_{1}(x)$ and $X_{2}(x)$ and force the matching of these two functions in a region far from the classical turning 
points $\left(x=-\infty\right.$ and $\left.x_{c}=\ln (-1 / E)\right)$. From the asymptotic behavior of the Bessel function of Eq. (29) and the Airy function of Eq. (33), we obtain:

$A \cos \left(\int_{-\infty}^{x} \sqrt{2 E e^{2 x}+2 e^{x}} d x-\frac{\pi}{4}\right)=B \cos \left(-\int_{x}^{x_{c}} \sqrt{2 E e^{2 x}+2 e^{x}} d x+\frac{\pi}{4}\right)$,

which implies:

$$
\begin{gathered}
\int_{-\infty}^{x} \sqrt{2 E e^{2 x}+2 e^{x}} d x-\frac{\pi}{4}=-\int_{x}^{x_{c}} \sqrt{2 E e^{2 x}+2 e^{x}} d x+\frac{\pi}{4}+n \pi \\
\int_{-\infty}^{x_{c}} \sqrt{2 E e^{2 x}+2 e^{x}} d x \quad=\pi\left(n+\frac{1}{2}\right),
\end{gathered}
$$

with $n=0,1,2, \ldots$, and $A_{n}=(-1)^{n} B_{n}$. This result is known as semiclassical quantisation conditions $[27,28]$, and is the same relation one would find with the usual JWKB approach. Solving this last integral explicitly yields:

$$
\frac{\pi}{\sqrt{-2 E}}=\pi\left(n+\frac{1}{2}\right) \Leftrightarrow E=-\frac{1}{2\left(n+\frac{1}{2}\right)^{2}},
$$

which exactly reproduces the energy levels of the 2D Hydrogen atom [29].

\section{Application to two-dimensional excitons}

In this section we apply the methods previously developed to the study of excitons in hexagonal Boron Nitride (hBN). We solve the Wannier equation, essentially a Schrodinger equation and find the binding energies and the wave functions of the states of zero angular momentum (the calculation for finite values of angular momentum follows the same approach; we do not pursue this path because these type of excitons are hardly observed). As we will see the results are in excellent agreement with those obtained using a more complex method based on the expansion of the wave function in terms of gaussians and diagonalizing a large matrix. The method of Miller and Good allows us to obtain quantitatively accurate results almost instantaneously.

To start calculations in this section, we introduce the Wannier equation, the equivalent to the Schrodinger equation when dealing with excitons, in S.I. units:

$$
\nabla^{2} \psi(r, \theta)+\frac{2 \mu}{\hbar^{2}}[E-V(r)] \psi(r, \theta)=0,
$$

where $\mu$ is the reduced mass of the electron-hole system and $V(r)$ is the RytovaKeldysh potential:

$$
V(r)=-\frac{e^{2}}{4 \pi \epsilon_{0}} \frac{\pi}{2} \frac{1}{r_{0}}\left[\mathbf{H}_{0}\left(\frac{\kappa r}{r_{0}}\right)-Y_{0}\left(\frac{\kappa r}{r_{0}}\right)\right],
$$

with $r_{0}$ a screening parameter characteristic of the 2D material and $\kappa$ the mean dielectric constant of the media above and below the $\mathrm{hBN}$ monolayer 
(in our case we take them to be air and quartz, respectively); $\mathbf{H}_{0}$ is the Struve function and $Y_{0}$ the Bessel function of the second kind. This potential is the solution of the Poisson equation for a thin film embedded in a medium (see [20] and [21]). Contrary to the previous section, we write this equation in S.I. units to allow us to compare our results with others present in the literature [18].

To solve this equation, an analogous procedure to the one employed in the previous section to solve the Schrodinger equation for the 2D Hydrogen atom is used; the only difference appears in the change of the Coulomb potential to the Rytova-Keldysh potential. The differential equation, after the Langer transformation, reads:

$$
X^{\prime \prime}(x)+\left(\frac{2 \mu}{\hbar^{2}} E e^{2 x}-\frac{2 \mu}{\hbar^{2}} V\left(e^{x}\right) e^{2 x}\right) X(x)=0,
$$

From this point onward all the results derive directly from the ones obtained in the previous section, substituting the terms inside the appropriate square roots by the term inside the parenthesis on the left and side of Eq. (43). Contrary to the Coulomb potential, where analytical expressions were found for every integral, the Rytova-Keldysh, due to its increased complexity (represented in terms of special functions), requires the integrals to be computed numerically (this is, however, an easy task).

The energies are obtained from the quantization condition:

$$
\int_{-\infty}^{x_{c}} \sqrt{E e^{2 x}-V\left(e^{x}\right) e^{2 x}} d x=\pi\left(n+\frac{1}{2}\right) \sqrt{\frac{\hbar^{2}}{2 m}}
$$

with $x_{c}$ given by $E-V\left(e^{x_{c}}\right)=0$. The results found for the binding energies of the $2 \mathrm{D}$ exciton in $\mathrm{hBN}$ are shown in Table 1 . There, we compare the energies found using the Miller and Good approach with the ones obtained in a previous work, where a semi-analytical method involving the diagonalization of a large matrix was used. The results here obtained agree quite well (less than 10\% difference) with those published previously in the literature [18]. Moreover, we observe that the results become increasingly more accurate as the principal quantum number increases, as was expected in any semiclassical description of a quantum problem (Bohr correspondence principle).

Finally, in Figure 1, we present the radial wave functions of the first three $s$-states, and compare them with the ones obtained in Ref. [18]; all of the three wave functions present a good agreement with the ones from the published literature. It is worth noting that the Miller and Good wave function does not diverge at the classical turning point, contrary to what would happen if the standard JWKB frame of work was employed. Moreover the calculation for both the binding energies and wave functions runs in an ordinary laptop in just few seconds. 


\begin{tabular}{cccc}
\hline & Miller and Good & Ref.[18] & Relative Difference \\
\hline \hline$n=1$ & -0.911 & -0.992 & $8.1 \%$ \\
\hline$n=2$ & -0.248 & -0.274 & $9.4 \%$ \\
\hline$n=3$ & -0.116 & -0.126 & $8.3 \%$ \\
\hline$n=4$ & -0.067 & -0.072 & $7.1 \%$ \\
\hline
\end{tabular}

Table 1 Binding energies of the first four $s$-states obtained using the Miller and Good method with the Rytova-Keldysh potential. Also presented are the energies obtained in Ref. [18]. The results computed in this work show a good agreement with the ones from the literature with increasing accuracy as the principal quantum number increases. All the energies are presented in $\mathrm{eV}$. To compute these energies an $r_{0}=10 \AA$ and $\kappa=2.4$ were used in the Rytova-Keldysh potential. The reduced mass was considered to be $\mu=m_{0} / 2$, with $m_{0}$ the bare electron mass.
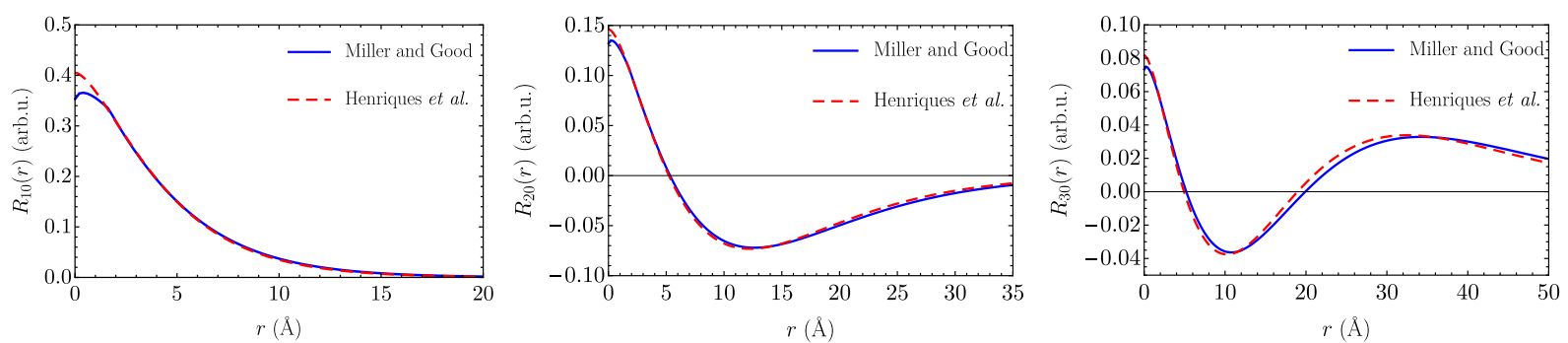

Fig. 1 First three $s$-states obtained using the Miller and Good method with the RytovaKeldysh potential compared with the ones obtained in the literature using a numerically exact method [18]. A remarkable good agreement between the semiclassical calculation and the numerically exact method is clearly seen.

\section{Conclusions}

In this paper we have revisited the uniform method of Miller and Good used to obtain continuous wave functions through all the real axis of an arbitrary one-dimensional potential. The method can cope with any state of angular momentum, with the zero angular momentum case being the most delicate (the one we treated in this paper). We have applied the method to the solution of Wannier equation in two-dimension and found a good agreement for both the binding energies and wave functions of excitons in hBN when compared to other computationally more demanding methods. This simplicity of our method allows one to study the dependence of the binding energy with different parameters of the system with little effort. In the case of the $2 \mathrm{D}$ Coulomb potential a full analytical solution is possible, which is expected since the original problem also has an exact solution. For the Rytova-Keldysh potential no exact solution is available, but the Miller-Good method provides a simple and accurate approximation to the wave functions and binding energies of this potential. As expected from any semiclassical method, the results are progressively better for higher quantum numbers. Despite this, we still found a good result to the binding energy of the ground state of the exciton. Although we have chosen a particularly simple material as a text bed for the Miller and 
Good approach, the method can easily be extended to other systems, such has excitons in transition metal dichalcogenides.

Acknowledgements The authors acknowledge Mike Belsley for carefully reading the manuscript and for comments that allowed to improve the overall presentation. N.M.R.P. acknowledges support from the European Commission through the project "Graphene-Driven Revolutions in ICT and Beyond" (Ref. No. 881603 - core 3), and the Portuguese Foundation for Science and Technology (FCT) in the framework of the Strategic Financing UID/FIS/04650/2019. In addition, N. M. R. P. acknowledges COMPETE2020, PORTUGAL2020, FEDER and the Portuguese Foundation for Science and Technology (FCT) through projects POCI- 010145-FEDER-028114, POCI-01-0145-FEDER-029265, PTDC/NAN-OPT/29265/2017, and POCI-01-0145-FEDER-02888.

\section{References}

1. H. Jeffreys, Proceedings of the London Mathematical Society 2(1), 428 (1923)

2. D. Gough, Astronomische Nachrichten 328(3-4), 273 (2007)

3. R. Blumel, Advanced quantum mechanics: the classical-quantum connection (Jones \& Bartlett Publishers, 2011)

4. M. Vandyck, Eur. J. Phys. 12(3), 112 (1991)

5. S.K. Adhikari, M.S. Hussein, Am. J. Phys. 76(12), 1108 (2008)

6. M.S. Child, Semiclassical mechanics with molecular applications (Oxford University Press, USA, 2014)

7. M.V. Berry, K. Mount, Rep. Prog. Phys. 35(1), 315 (1972)

8. A. Amthong, Eur. J. Phys. 35(6), 065009 (2014)

9. M. Arzamasovs, B. Liu, Eur. J. Phys. 38(6), 065405 (2017)

10. S.C. Miller Jr, R. Good Jr, Phys. Rev. 91(1), 174 (1953)

11. S. Miller Jr, Phys. Rev. 94(5), 1345 (1954)

12. R. Good Jr, Phys. Rev. 90(1), 131 (1953)

13. M. Berry, A.O. de Almeida, J. Phys. A 6(10), 1451 (1973)

14. G. Wang, A. Chernikov, M.M. Glazov, T.F. Heinz, X. Marie, T. Amand, B. Urbaszek, Rev. Mod. Phys. 90, 021001 (2018)

15. A. Chaves, R. Ribeiro, T. Frederico, N. Peres, 2D Materials 4(2), 025086 (2017)

16. K. Zhang, Y. Feng, F. Wang, Z. Yang, J. Wang, Journal of Materials Chemistry C 5(46), 11992 (2017)

17. J.D. Caldwell, I. Aharonovich, G. Cassabois, J.H. Edgar, B. Gil, D. Basov, Nature Reviews Materials 4(8), 552 (2019)

18. J. Henriques, G. Ventura, C. Fernandes, N. Peres, J. Phys.: Cond. Matt. 32(2), 025304 (2020)

19. S. Das, J.A. Robinson, M. Dubey, H. Terrones, M. Terrones, Ann. Rev. Mat. Res. 45 $1(2015)$

20. S. Rytova, Moscow University Physics Bulletin 22(30) (1967)

21. L. Keldysh, Sov. J. Exp. and Theor. Phys. Lett. 29, 658 (1979)

22. T. Galvani, F. Paleari, H.P.C. Miranda, A. Molina-Sánchez, L. Wirtz, S. Latil, H. Amara, F.m.c. Ducastelle, Phys. Rev. B 94, 125303 (2016)

23. A.K. Ghatak, R. Gallawa, I. Goyal, NIST 92, 20427 (1991)

24. I. Goyal, R. Gallawa, A. Ghatak, Journal of electromagnetic waves and applications $\mathbf{5}(6), 623(1991)$

25. R.E. Langer, Phys. Rev. 51(8), 669 (1937)

26. J.P. Dahl, W.P. Schleich, The Journal of Physical Chemistry A 108(41), 8713 (2004)

27. A. Sinha, R. Roychoudhury, Y. Varshni, Physica B: Cond. Matt. 325, 214 (2003)

28. M.V. Berry, K. Burke, Eur. J. Phys. 40(6), 065403 (2019)

29. X. Yang, S. Guo, F. Chan, K. Wong, W. Ching, Phys. Rev. A 43(3), 1186 (1991)

30. J.S. Cohen, The J. Chem. Phys. 68(4), 1841 (1978)

31. J.R. Taylor, Il Nuovo Cimento B (1971-1996) 23(2), 313 (1974)

32. S.K. Adhikari, Am. J. Phys. 54(4), 362 (1986)

33. G. Barton, Proceedings of the Royal Society of London. A. Mathematical and Physical Sciences 388(1795), 419 (1983)

34. M. Rosen, D.R. Yennie, J. Math. Phys. 5(11), 1505 (1964) 